Washington University School of Medicine Digital Commons@Becker

Open Access Publications

2013

\title{
Effect of daily chlorhexidine bathing on hospital-acquired infection
}

\author{
Michael W. Climo \\ Hunter Holmes McGuire Veterans Affairs Medical Center \\ Deborah S. Yokoe \\ Harvard Medical School \\ David K. Warren \\ Washington University School of Medicine in St. Louis \\ Trish M. Perl \\ John Hopkins University \\ Maureen Bolon \\ Northerwestern University
}

See next page for additional authors

Follow this and additional works at: https://digitalcommons.wustl.edu/open_access_pubs

Please let us know how this document benefits you.

\section{Recommended Citation}

Climo, Michael W.; Yokoe, Deborah S.; Warren, David K.; Perl, Trish M.; Bolon, Maureen; Herwaldt, Loreen A.; Weinstein, Robert A.; Sepkowitz, Kent A.; Jernigan, John A.; Sanogo, Kakotan; and Wong, Edward S., "Effect of daily chlorhexidine bathing on hospital-acquired infection." The New England Journal of Medicine. 368, 6. 533-542. (2013).

https://digitalcommons.wustl.edu/open_access_pubs/2575

This Open Access Publication is brought to you for free and open access by Digital Commons@Becker. It has been accepted for inclusion in Open Access Publications by an authorized administrator of Digital Commons@Becker. For more information, please contact vanam@wustl.edu. 


\section{Authors}

Michael W. Climo, Deborah S. Yokoe, David K. Warren, Trish M. Perl, Maureen Bolon, Loreen A. Herwaldt, Robert A. Weinstein, Kent A. Sepkowitz, John A. Jernigan, Kakotan Sanogo, and Edward S. Wong 


\section{Effect of Daily Chlorhexidine Bathing on Hospital-Acquired Infection}

\author{
Michael W. Climo, M.D., Deborah S. Yokoe, M.D., M.P.H., David K. Warren, M.D., \\ Trish M. Perl, M.D., Maureen Bolon, M.D., Loreen A. Herwaldt, M.D., \\ Robert A. Weinstein, M.D., Kent A. Sepkowitz, M.D., John A. Jernigan, M.D., \\ Kakotan Sanogo, M.S., and Edward S. Wong, M.D.
}

A BSTRACT

\section{BACKGROUND}

Results of previous single-center, observational studies suggest that daily bathing of patients with chlorhexidine may prevent hospital-acquired bloodstream infections and the acquisition of multidrug-resistant organisms (MDROs).

\section{METHODS}

We conducted a multicenter, cluster-randomized, nonblinded crossover trial to evaluate the effect of daily bathing with chlorhexidine-impregnated washcloths on the acquisition of MDROs and the incidence of hospital-acquired bloodstream infections. Nine intensive care and bone marrow transplantation units in six hospitals were randomly assigned to bathe patients either with no-rinse $2 \%$ chlorhexidineimpregnated washcloths or with nonantimicrobial washcloths for a 6-month period, exchanged for the alternate product during the subsequent 6 months. The incidence rates of acquisition of MDROs and the rates of hospital-acquired bloodstream infections were compared between the two periods by means of Poisson regression analysis.

\section{RESULTS}

A total of 7727 patients were enrolled during the study. The overall rate of MDRO acquisition was 5.10 cases per 1000 patient-days with chlorhexidine bathing versus 6.60 cases per 1000 patient-days with nonantimicrobial washcloths $(\mathrm{P}=0.03)$, the equivalent of a $23 \%$ lower rate with chlorhexidine bathing. The overall rate of hospital-acquired bloodstream infections was 4.78 cases per 1000 patient-days with chlorhexidine bathing versus 6.60 cases per 1000 patient-days with nonantimicrobial washcloths $(\mathrm{P}=0.007)$, a $28 \%$ lower rate with chlorhexidine-impregnated washcloths. No serious skin reactions were noted during either study period.

\section{CONCLUSIONS}

Daily bathing with chlorhexidine-impregnated washcloths significantly reduced the risks of acquisition of MDROs and development of hospital-acquired bloodstream infections. (Funded by the Centers for Disease Control and Prevention and Sage Products; ClinicalTrials.gov number, NCT00502476.)
From the Hunter Holmes McGuire Veterans Affairs Medical Center (M.W.C., E.S.W.) and the Virginia Commonwealth University Medical Center (M.W.C., K.S., E.S.W.), Richmond; Brigham and Women's Hospital and Harvard Medical School, Boston (D.S.Y.); Washington University School of Medicine, St. Louis (D.K.W.); Johns Hopkins University, Baltimore (T.M.P.); Northwestern University (M.B.) and Cook County Health and Hospitals System (R.A.W.), Chicago; lowa University Hospital, lowa City (L.A.H.); Memorial Sloan-Kettering Cancer Center, New York (K.A.S.); and the Prevention Epicenters Program, Centers for Disease Control and Prevention, Atlanta (J.A.J.). Address reprint requests to Dr. Climo at the McGuire Veterans Affairs Medical Center, 1201 Broad Rock Blvd., Section 111-C, Richmond, VA 23249, or at michael.climo@va.gov.

This article was updated on May 23, 2013, at NEJM.org.

N EnglJ Med 2013;368:533-42. DOI: 10.1056/NEJMoall13849

Copyright (C) 2013 Massachusetts Medical Society. 
M ULTIDRUG-RESISTANT ORGANISMS (MDROs), including methicillin-resistant Staphylococcus aureus (MRSA) and vancomycin-resistant enterococcus (VRE), have become endemic in many acute care and longterm care facilities. ${ }^{1-5}$ Infections with these organisms are often difficult to treat, owing to a dwindling armamentarium of active antimicrobial agents. The Centers for Disease Control and Prevention (CDC) has promulgated a variety of strategies, including hand hygiene and the use of isolation precautions, to limit the spread of these organisms among patients, but these strategies require consistent adherence to practices by large numbers of health care personnel during frequent patient encounters and can be difficult to sustain. ${ }^{6}$ In addition, health care-associated infections involving these and other microorganisms $\mathrm{s}^{7,8}$ are associated with considerable morbidity and mortality and with substantial excess costs that, in some cases, are no longer reimbursed by third-party payers, including the Centers for Medicare and Medicaid Services. ${ }^{9,10}$

Targeted interventions, particularly in intensive care units (ICUs), can substantially reduce the risk of hospital-acquired bloodstream infections associated with the use of central venous catheters. Several large studies have shown that improving catheter-insertion processes, including standardizing insertion-site antisepsis with the use of chlorhexidine-containing products, can decrease the risk of infection. ${ }^{11-13}$ However, the use of antiseptic agents for patient bathing is currently considered controversial.

Chlorhexidine gluconate is an antiseptic agent that has broad-spectrum activity against many organisms, including $S$. aureus and enterococcus species. Unlike many other antiseptics, chlorhexidine has residual antibacterial activity, which may decrease the microbial burden on patients' skin and prevent secondary environmental contamination. Vernon et al. found that daily bathing with chlorhexidine-impregnated cloths decreased the number of VRE colonies on skin by $2.5 \mathrm{log}$, as compared with bathing with soap and water, as well as decreasing VRE contamination of health care workers' hands by $40 \%$ and environmental surfaces by $30 \% .{ }^{14}$ By controlling the source, these investigators reduced the rate of acquisition of VRE among patients by $66 \%$.
Because hospital-acquired bloodstream infections often result from the ingress of skin organisms into the bloodstream along vascular catheters or other breaks in skin integrity, skin decontamination could theoretically also decrease the risk of infection. Bleasdale et al. found that daily bathing with $2 \%$ chlorhexidine-impregnated washcloths reduced the incidence of primary bloodstream infections by 60\%.15 Our previous observational study evaluating bathing with chlorhexidine in six ICUs showed a $66 \%$ reduction in VRE bacteremia. ${ }^{16}$ Previous studies of bathing with chlorhexidine have been primarily single-center, before-and-after, observational studies, with limited general applicability of results. We therefore conducted a multicenter, randomized trial to evaluate the usefulness of bathing with chlorhexidine to reduce the risks of MDRO acquisition and hospital-acquired bloodstream infection among patients at high risk for health care-associated infections.

METHODS

\section{STUDY DESIGN}

We performed a cluster-randomized, crossover study involving patients hospitalized in six ICUs or bone marrow transplantation units between August 2007 and February 2009. Units were randomly assigned to perform daily bathing of patients with either nonantimicrobial washcloths (Comfort Bath, Sage Products) (control) or washcloths impregnated with $2 \%$ chlorhexidine gluconate (2\% Chlorhexidine Gluconate Cloth Patient Preoperative Skin Preparation, Sage Products) (intervention) during the initial 6-month study period, followed by daily bathing with the alternate product during the second 6-month period.

Bathing was completed according to the manufacturer's instructions. In brief, washcloths were used in sequential order to rinse all body surfaces, with the exception of the face during bathing with the $2 \%$ chlorhexidine-impregnated cloths in order to avoid exposure of the mucous membranes of the eyes and mouth. There was no washout period in the transition to the new product. Infections and MDRO acquisitions were monitored for 2 days after the transition and assigned to the previous bathing treatment if they occurred within that time period. 
The order in which units were assigned to the control or intervention period was stratified according to unit type and facility. The nine participating units were divided into two groups. Group 1 (five units) started with bathing with the chlorhexidine-impregnated washcloths, followed by bathing with nonantimicrobial washcloths. Group 2 (four units) started with bathing with nonantimicrobial washcloths, followed by bathing with the chlorhexidine-impregnated washcloths. The investigators and clinical staff were aware of the use of the control or intervention bathing product.

Before the study was initiated, nurses were instructed on the proper techniques for bathing patients with both washcloth products. Skin-care products that were not compatible with chlorhexidine were eliminated before the study began. Nursing personnel monitored patients for skin reactions and reported them to the investigators, who graded skin reactions on a scale of 1 to 4 (with higher numbers indicating greater severity) and determined whether the reactions were attributable to bathing (for details of the assessments and scales, see the Supplementary Appendix, available with the full text of this article at NEJM.org).

All units performed active surveillance testing for MRSA and VRE throughout the study period. Unit staff obtained swabs from the nares (for MRSA) and perirectal area (for VRE) from patients up to 48 hours after admission to the unit and on discharge from the unit. The microbiology laboratory at each institution processed surveillance specimens using either standard culturebased or molecular-based (polymerase chain reaction) identification of MRSA and VRE. All patients found to be colonized or infected with MRSA or VRE were placed on contact precautions once test results became available. Patients with a history of MRSA or VRE colonization or infection were placed on contact precautions at the time of admission.

Each participating unit submitted at least 10 separate MRSA and VRE isolates obtained from patients to the coordinating center each month for chlorhexidine susceptibility testing. Susceptibility testing was completed by means of the agar dilution method, with chlorhexidine concentrations ranging from 0.1 to $1024.0 \mu \mathrm{g}$ per

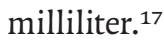

\section{STUDY OVERSIGHT}

Sage Products supplied the chlorhexidine-impregnated and nonantimicrobial washcloths to participating units for the duration of the study, provided technical and educational support, and participated in weekly teleconferences with the study group during the conduct of the study but was not involved in the study design, the data analysis, or the preparation of the manuscript. Approval of the study protocol was obtained from institutional review boards at the study centers and the CDC. Waiver of written informed consent was obtained at each institution, owing to the minimal-risk nature of the study. Patients who declined to participate were not bathed with chlorhexidine-impregnated washcloths. All authors vouch for the completeness and accuracy of the data presented and for the fidelity of this report to the study protocol, which is available at NEJM.org.

\section{DEFINITIONS}

Incident and prevalent cases of MRSA or VRE colonization or infection were classified as previously described. ${ }^{16}$ Bloodstream infections were identified with the use of National Healthcare Safety Network definitions. ${ }^{18}$ Hospital-acquired bloodstream infections were defined as bloodstream infections detected more than 48 hours after admission to the unit. Primary bloodstream infections were defined as hospital-acquired bloodstream infections detected more than 48 hours after admission to the unit without an attributable secondary source of infection. Central-catheterassociated bloodstream infections were defined as primary bloodstream infections in patients with at least one central venous catheter in place within 48 hours before detection of the infection.

\section{TREATMENT INTERRUPTION}

On June 28, 2008, Sage Products initiated a nationwide recall of the $2 \%$ chlorhexidine-impregnated washcloths, because of Burkholderia cepacia contamination of some product lots. Units using the chlorhexidine product at the time of the recall were switched to the nonantimicrobial washcloths, and the institutional review boards were immediately notified. After remediation and approval by the institutional review boards, use of the chlorhexidine product was resumed. Data from units that had been assigned to use the chlorhexidine-based 


\begin{tabular}{|c|c|c|c|c|c|c|c|}
\hline Hospital & Unit & $\begin{array}{l}\text { Mean No. } \\
\text { of Monthly } \\
\text { Admissions } \\
\text { numbe }\end{array}$ & $\begin{array}{l}\text { Mean No. } \\
\text { of Monthly } \\
\text { Patient-Days } \\
\text { (range) }\end{array}$ & $\begin{array}{c}\text { Mean Length } \\
\text { of Stay } \\
\text { days }\end{array}$ & $\begin{array}{c}\text { MRSA } \\
\text { Prevalence } \\
\text { percent o }\end{array}$ & $\begin{array}{l}\text { VRE } \\
\text { Prevalence } \\
\text { dmissions }\end{array}$ & $\begin{array}{c}\text { Baseline Rate of } \\
\text { Primary Bloodstream } \\
\text { Infections' } \\
\text { no. } / 1000 \text { patient-days }\end{array}$ \\
\hline \multicolumn{8}{|l|}{ Group 1} \\
\hline A & MICU & $123.8(114-142)$ & $692.3(504-773)$ & 5.6 & 11.0 & 21.0 & 8.1 \\
\hline C & SICU & $46.3(31-59)$ & $285.7(251-314)$ & 6.2 & 11.4 & 4.3 & 9.6 \\
\hline D & SICU 2 & $51.6(32-71)$ & $285.7(227-338)$ & 5.5 & 4.4 & 2.8 & 0 \\
\hline$E$ & CSICU & $85.3(80-100)$ & $425.9(375-486)$ & 5.0 & 6.6 & 8.3 & 0.4 \\
\hline $\mathrm{F}$ & ВMT & $41.8(32-58)$ & $786.3(725-858)$ & 18.8 & 2.4 & 21.6 & 5.5 \\
\hline \multicolumn{8}{|l|}{ Group 2} \\
\hline B & MICU & $111.6(98-126)$ & $598.8(449-641)$ & 5.4 & 21.8 & 21.0 & 3.1 \\
\hline C & MICU-CCU & $55.8(43-73)$ & $299.1(211-345)$ & 5.4 & 16.1 & 9.7 & 8.5 \\
\hline D & SICU 1 & $62.3(47-76)$ & $316.3(266-356)$ & 5.1 & 10.8 & 8.2 & 2.2 \\
\hline$E$ & MICU & $72.7(56-88)$ & $467.1(404-525)$ & 6.4 & 23.3 & 27.9 & 8.7 \\
\hline
\end{tabular}

* Group 1 used chlorhexidine-impregnated washcloths during the first 6-month period and nonantimicrobial washcloths during the second 6-month period, and group 2 did the reverse. BMT denotes bone marrow transplantation unit, CSICU cardiac surgery intensive care unit, MICU medical intensive care unit, MICU-CCU combined medical intensive care unit and coronary care unit, MRSA methicillin-resistant Staphylococcus aureus, SICU surgical intensive care unit, and VRE vancomycin-resistant enterococcus.

$\dagger$ The baseline rate of primary bloodstream infections was defined as the number of new cases among eligible patients during the control period.

washcloths during the recall period were censored from the final analysis.

\section{STATISTICAL ANALYSIS}

We evaluated changes in the mean rates of MRSA and VRE acquisition and hospital-acquired bloodstream infections. We tested the null hypothesis that the rates during the control period equaled the rates during the intervention period, using the PROC GENMOD procedure in SAS software, version 8.2 (SAS Institute), to fit a Poisson regression model that accounted for monthly prevalence of MRSA and VRE colonizations or infections in each unit as possible confounders.

We used a Cox proportional-hazards regression model to compare the time from admission until the first primary bloodstream infection between the control and intervention periods. We calculated the survival time as the interval between admission and discharge from the study unit for those patients who did not acquire a primary bloodstream infection and as the interval between admission and the first positive culture for patients with a primary bloodstream infection.

We examined the effect of the following unit characteristics on the rates of primary bloodstream infections: unit size, unit type, mean length of stay, rate of use of central venous catheters, median patient age, distribution of patient sex, monthly rate of incident MRSA colonizations or infections, monthly rate of incident VRE colonizations or infections, rate of prevalent MRSA colonization or infection at the time of admission, and rate of prevalent VRE colonization or infection at the time of admission. We compared changes in the rates of incident primary bloodstream infections between the control period and the intervention period. Continuous variables were examined with the use of two-sample t-tests and linear regression modeling, and categorical variables were examined by means of Fisher's exact test.

\section{RESULTS}

\section{CHARACTERISTICS OF THE STUDY UNITS}

Twelve units from seven hospitals were recruited to participate in the planned 12-month study. One unit withdrew from the study, and two units were eliminated from the analysis because of low compliance with the study protocol. The final nine study units included medical, coronary care, surgical, and cardiac surgery ICUs and one bone marrow transplantation unit (Table 1$)$. Only $8(0.1 \%)$ 


\begin{tabular}{|c|c|c|c|}
\hline Variable & Intervention Period & Control Period & P Value \\
\hline No. of admissions & 3970 & 3842 & 0.32 \\
\hline Total days of care & 24,902 & 24,983 & 0.85 \\
\hline Central-catheter use (days) & 13,425 & 13,049 & 0.14 \\
\hline Mean length of stay (days) & 6.4 & 6.4 & 0.53 \\
\hline MRSA prevalence (\%) & 13.8 & 12.8 & 0.14 \\
\hline VRE prevalence (\%) & 16.3 & 15.1 & 0.24 \\
\hline \multicolumn{4}{|l|}{ MDRO acquisition } \\
\hline No. of infections & 127 & 165 & 0.03 \\
\hline Incidence rate (no./1000 patient-days) & 5.10 & 6.60 & \\
\hline \multicolumn{4}{|l|}{ VRE acquisition } \\
\hline No. of infections & 80 & 107 & 0.05 \\
\hline Incidence rate (no./1000 patient-days) & 3.21 & 4.28 & \\
\hline \multicolumn{4}{|l|}{ MRSA acquisition } \\
\hline No. of infections & 47 & 58 & 0.29 \\
\hline Incidence rate (no./1000 patient-days) & 1.89 & 2.32 & \\
\hline \multicolumn{4}{|l|}{ Hospital-acquired bloodstream infection } \\
\hline No. of infections & 119 & 165 & 0.007 \\
\hline Incidence rate (no./1000 patient-days) & 4.78 & 6.60 & \\
\hline \multicolumn{4}{|l|}{ Primary bloodstream infection } \\
\hline No. of infections & 90 & 131 & 0.006 \\
\hline Incidence rate (no./1000 patient-days) & 3.61 & 5.24 & \\
\hline \multicolumn{4}{|c|}{ Central-catheter-associated bloodstream infection } \\
\hline No. of infections & 21 & 43 & 0.004 \\
\hline Incidence rate (no./1000 catheter-days) & 1.55 & 3.30 & \\
\hline \multicolumn{4}{|l|}{ Secondary bloodstream infection } \\
\hline No. of infections & 29 & 34 & 0.45 \\
\hline Incidence rate (no./1000 patient-days) & 1.20 & 1.40 & \\
\hline
\end{tabular}

* Prevalence was defined as the total number of prevalent cases per 100 patients admitted to the study unit. The incidence rate was defined as the total number of acquired cases among eligible patients.

of 7735 patients admitted to the participating units declined to participate in the study, and data from all 7727 patients who agreed to participate were included in an intention-to-treat analysis.

\section{ACQUISITION OF MRSA AND VRE}

During the control period, when nonantimicrobial cloths were used, 165 new cases of MRSA or VRE acquisition were detected, as compared with 127 during the periods of bathing with chlorhexidine. The overall rate of MRSA or VRE acquisition was $23 \%$ lower during the intervention period (5.10 vs.
6.60 cases per 1000 patient-days, $\mathrm{P}=0.03$ ) (Table 2). Reductions in the incidence of VRE and MRSA acquisition were unrelated to the monthly prevalence of either MRSA or VRE colonization or infection.

The overall rate of VRE acquisition was 25\% lower during the intervention period than during the control period (3.21 vs. 4.28 cases per 1000 patient-days, $\mathrm{P}=0.05)$. The overall rate of MRSA acquisition was $19 \%$ lower during the intervention period than during the control period, but this difference was not significant (1.89 vs. 2.32 cases per 1000 patient-days, $\mathrm{P}=0.29)$. 


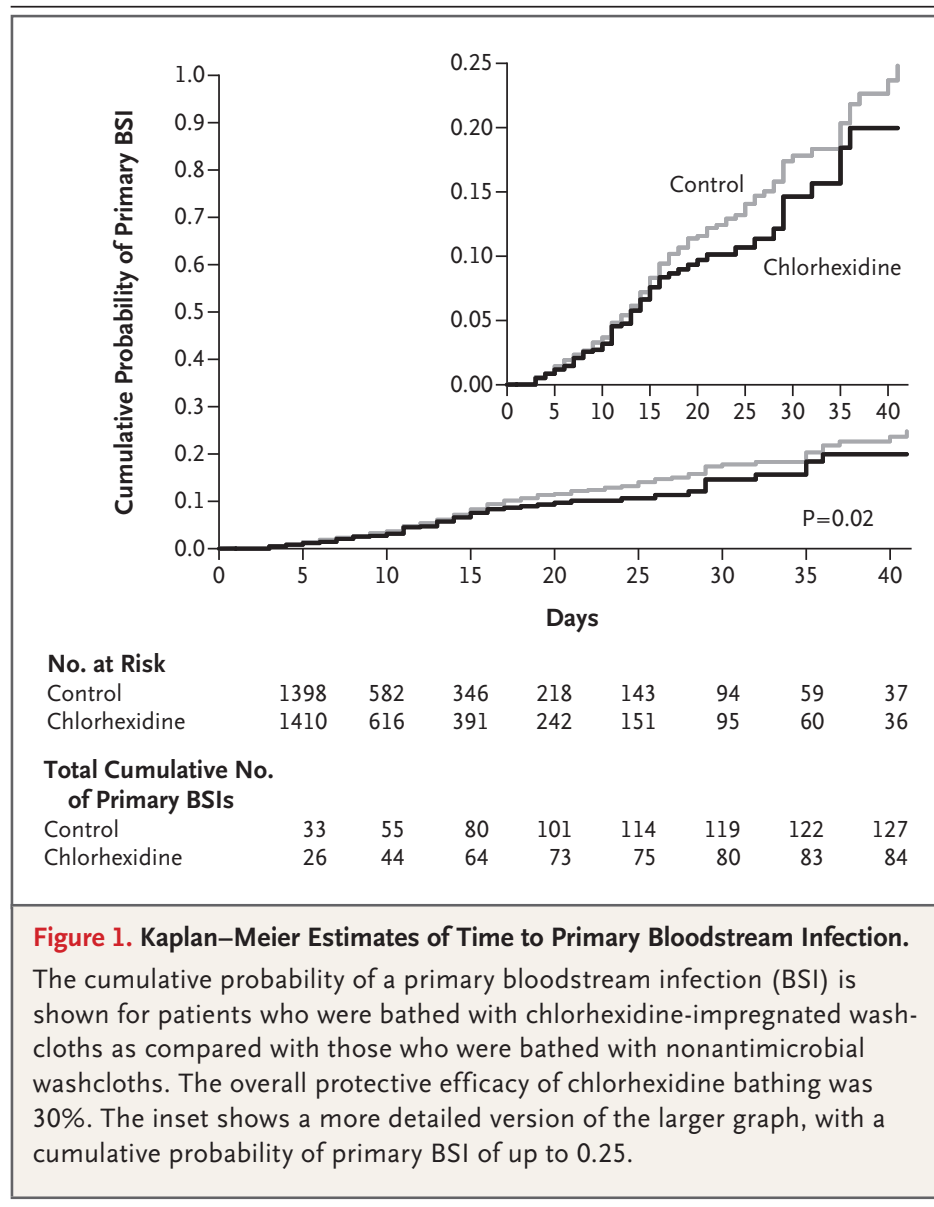

BLOODSTREAM INFECTIONS

Overall, 165 hospital-acquired bloodstream infections were detected in patients during the control period, as compared with 119 during the intervention period. The rate of hospital-acquired bloodstream infections was $28 \%$ lower during the intervention period than during the control period ( 4.78 vs. 6.60 cases per 1000 patient-days, $\mathrm{P}=0.007$ ) (Table 2). This finding reflected the $31 \%$ lower rate of primary bloodstream infections during the intervention period, as compared with the control period (3.61 vs. 5.24 cases per 1000 patient-days, $\mathrm{P}=0.006$ ). The rate of central-catheter-associated bloodstream infections was $53 \%$ lower during the intervention period than during the control period (1.55 vs. 3.30 cases per 1000 catheter-days, $\mathrm{P}=0.004)$. The rate of secondary bloodstream infections did not differ significantly between the intervention and control periods.

On the basis of the Cox proportional-hazards survival regression analysis, the risk of acquiring a primary bloodstream infection was significantly lower among patients bathed with chlorhexidine than among those bathed with the nonantimicrobial cloths $(\mathrm{P}=0.02)$ (Fig. 1). This effect was greater among patients with a longer length of stay in the unit. Among patients who were in the unit for more than 7 days, the relative risk of a primary bloodstream infection was 0.69 (95\% confidence interval [CI], 0.47 to 0.99 ) for patients bathed with chlorhexidine as compared with those bathed with the nonantimicrobial washcloths. Among patients who were in the unit for more than 14 days, the relative risk of a primary bloodstream infection was 0.51 (95\% CI, 0.30 to 0.87 ) among patients bathed with chlorhexidine as compared with those who were bathed with the nonantimicrobial washcloths.

Among the 221 primary bloodstream infections, the most common pathogens were staphylococci $(30 \%)$, gram-negative bacilli $(23 \%)$, enterococci (20\%), and fungi (13\%) (Table 3). The incidence rate of primary bloodstream infection caused by coagulase-negative staphylococci was $56 \%$ lower during the intervention period than during the control period ( 0.60 vs. 1.36 cases per 1000 patient-days, $\mathrm{P}=0.008$ ). Similarly, the incidence rate of primary bloodstream infection caused by fungi was $53 \%$ lower during the intervention period than during the control period, but this finding was not significant $(0.36$ vs. 0.76 cases per 1000 patient-days, $\mathrm{P}=0.06$ ).

The incidence of central-catheter-associated bloodstream infections was significantly lower during the intervention period than during the control period for infections involving grampositive organisms ( 0.89 vs. 1.76 cases per 1000 catheter-days, $\mathrm{P}=0.05$ ) and those involving fungi $(0.07$ vs. 0.77 cases per 1000 catheter-days, $\mathrm{P}<0.001)$. Overall, the incidence of central-catheter-associated fungal bloodstream infection was $90 \%$ lower during the intervention period than during the control period. Bathing with chlorhexidine was not associated with a significant reduction in the incidence of central-catheter-associated bloodstream infections involving gram-negative organisms or those involving VRE or MRSA, findings that are probably related to the low number of infections caused by these organisms.

Owing to concern that the interruption of treatment may have affected the observed outcomes, we performed an additional analysis of the incidence rates of bloodstream infections that in- 


\begin{tabular}{|c|c|c|c|c|c|}
\hline \multirow[t]{2}{*}{ Agent } & \multicolumn{2}{|c|}{ Intervention Period } & \multicolumn{2}{|c|}{ Control Period } & \multirow[t]{2}{*}{ P Value } \\
\hline & $\begin{array}{c}\text { No. of } \\
\text { Infections }\end{array}$ & Incidence Rate & $\begin{array}{c}\text { No. of } \\
\text { Infections }\end{array}$ & Incidence Rate & \\
\hline Staphylococci & 24 & 0.96 & 42 & 1.68 & 0.03 \\
\hline Staphylococcus aureus & 9 & 0.36 & 8 & 0.32 & 0.80 \\
\hline Coagulase-negative staphylococci & 15 & 0.60 & 34 & 1.36 & 0.008 \\
\hline Enterococci & 19 & 0.76 & 26 & 1.04 & 0.30 \\
\hline Enterococcus faecalis & 13 & 0.52 & 19 & 0.76 & 0.29 \\
\hline E. faecium & 6 & 0.24 & 6 & 0.24 & 1.00 \\
\hline Gram-negative bacilli & 23 & 0.92 & 27 & 1.08 & 0.58 \\
\hline Acinetobacter & 1 & 0.04 & 2 & 0.08 & 1.00 \\
\hline Escherichia & 8 & 0.32 & 6 & 0.24 & 0.52 \\
\hline Enterobacter & 2 & 0.08 & 8 & 0.32 & 0.06 \\
\hline Klebsiella & 5 & 0.20 & 5 & 0.20 & 1.00 \\
\hline Pseudomonas & 4 & 0.16 & 2 & 0.08 & 0.41 \\
\hline Serratia & 2 & 0.08 & 1 & 0.04 & 1.00 \\
\hline Stenotrophomonas & 0 & 0.00 & 1 & 0.04 & 1.00 \\
\hline Other & 1 & 0.04 & 2 & 0.08 & 1.00 \\
\hline Fungi & 9 & 0.36 & 19 & 0.76 & 0.06 \\
\hline Candida & 7 & 0.28 & 16 & 0.64 & 0.06 \\
\hline Other & 2 & 0.08 & 3 & 0.12 & 0.66 \\
\hline Polymicrobial organisms & 9 & 0.36 & 12 & 0.48 & 0.52 \\
\hline Other & 6 & 0.24 & 5 & 0.20 & 0.76 \\
\hline Total & 90 & 3.61 & 131 & 5.24 & 0.01 \\
\hline
\end{tabular}

$*$ The incidence rate was defined as the number of primary bloodstream infections per 1000 patient-days.

cluded those months when nonantimicrobial cloths were used by units affected by the national recall. The addition of data obtained during the 4 months of treatment interruption, when only nonantimicrobial bathing cloths were used, did not alter the analysis results. In this analysis, 58 months of use of nonantimicrobial cloths for bathing was compared with 54 months of use of the $2 \%$ chlorhexidine-impregnated cloths. The overall results remained the same. The overall incidence of hospital-acquired bloodstream infection was decreased in those months when chlorhexidine was in use, as compared with the use of the nonantimicrobial washcloths (4.78 vs. 6.32 cases per 1000 patient-days, $\mathrm{P}=0.02$ ).

\section{UNIT CHARACTERISTICS}

Reductions in rates of primary bloodstream infections were highest among medical ICUs. The rate of primary bloodstream infections in medi- cal ICUs was $40 \%$ lower during the intervention period than during the control period (3.98 vs. 6.62 cases per 1000 patient-days). In contrast, the rate of primary bloodstream infections in other units was $17 \%$ lower during the intervention period than during the control period (3.10 vs. 3.73 cases per 1000 patient-days) (Fig. 2). However, the observed reductions in the rate of primary bloodstream infections among medical ICUs were not significantly associated with the unit type. Other unit characteristics - unit size, mean length of stay, baseline rate of primary bloodstream infections, median age of patients, MRSA and VRE prevalence, catheter use, and sex distribution were not associated with changes in the rates of primary bloodstream infections.

\section{CHLORHEXIDINE SUSCEPTIBILITY}

We performed antimicrobial susceptibility testing on clinical isolates collected during the entire 


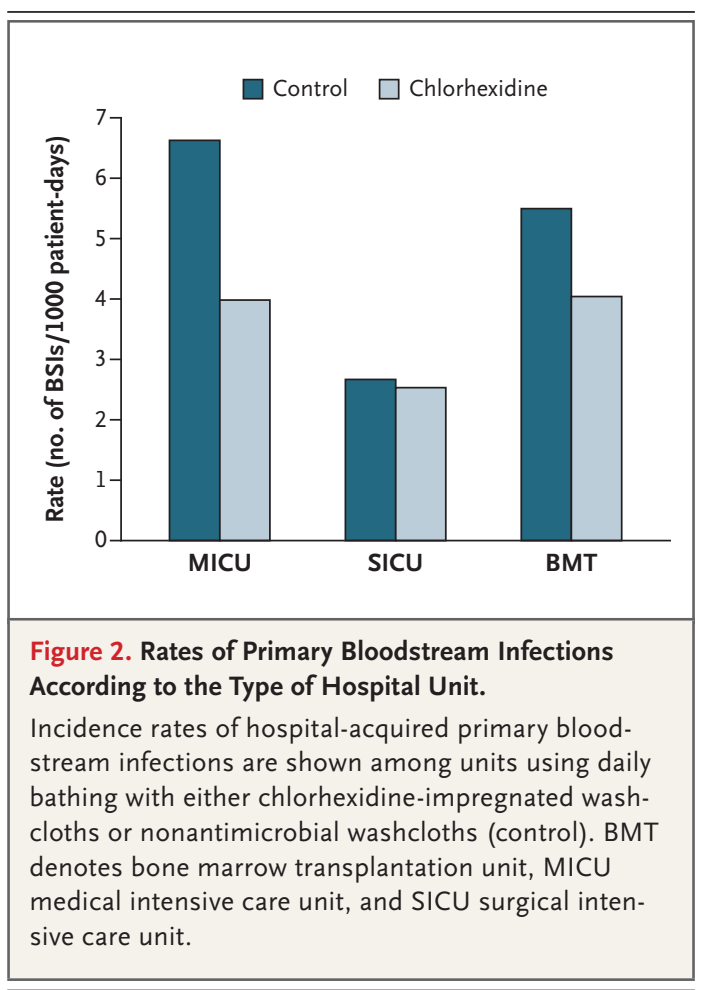

study period. We tested a total of 1106 isolates (713 MRSA and 393 VRE) for susceptibility to chlorhexidine by means of the agar dilution method. Chlorhexidine was slightly more active against MRSA isolates, with a minimum inhibitory concentration required to inhibit the growth of $90 \%$ of organisms of $4 \mu \mathrm{g}$ per milliliter, as compared with $8 \mu \mathrm{g}$ per milliliter for VRE isolates.

\section{ADVERSE REACTIONS}

The overall incidence of skin reactions among patients assigned to chlorhexidine bathing was $2.0 \%$ (78 of 3970 patients), as compared with $3.4 \%$ (130 of 3842) among those assigned to bathing with the control product. All 208 reported skin reactions were considered to be unrelated to the bathing intervention, and overall, $85 \%$ of the reactions were classified as mild to moderate (grade 1 or 2).

\section{DISCUSSION}

The findings of this multicenter, cluster-randomized study evaluating daily bathing with chlorhexidine support the results of previous single-center trials suggesting that bathing with chlorhexidine reduces the transmission of resistant organisms and the risk of hospital-acquired bloodstream infections among patients in ICUs and bone marrow transplantation units. ${ }^{14-16,19,20}$ In addition, the participation of facilities from different geographic regions in the United States supports the generalizability of these results to other academic medical centers.

Our results support the findings of Vernon et al. and Bleasdale et al. and suggest that bathing with chlorhexidine may be particularly effective in reducing the risk of bloodstream infections among patients in the ICU. ${ }^{14,15}$ In contrast to these previous studies, which involved a limited number of units, our multicenter design allowed a more robust examination of whether the reductions in rates of bloodstream infections were related to the type of unit. We found no significant interaction between the type of unit and the development of bloodstream infections, suggesting that bathing with chlorhexidine may be beneficial in many unit settings. In addition, bathing with chlorhexidine may be particularly beneficial for patients with long ICU stays.

Our study also had some unanticipated findings. First, as compared with bathing with the control product, bathing with chlorhexidine was associated with lower rates of central-catheterassociated fungal bloodstream infection. Previous studies have shown that bathing with chlorhexidine is associated with a lower risk of central-catheter-associated bloodstream infection involving gram-positive cocci, ${ }^{14-16}$ but reduced rates of fungemia have not been reported. Chlorhexidine has biphasic fungicidal activity, ${ }^{21}$ but topical use has not been suggested as a possible intervention to reduce the incidence of fungemia among patients with indwelling central catheters. Previous efforts to reduce the incidence of fungemia have relied mostly on systemic antifungal prophylaxis, which can increase the incidence of antifungal resistance among fungal isolates. ${ }^{22}$ If our results are confirmed, topical use of chlorhexidine could be added to strategies to prevent fungal infections.

Bathing with chlorhexidine was associated with significant reductions in the incidence of gram-positive bacteremias, findings that are similar to the results of previous studies. The majority of these reductions were related to the reductions in the incidence of infections involving coagulase-negative staphylococci. Despite the overall reduction in the acquisition of MRSA 
and VRE, significant reductions in the incidence of MRSA and VRE bacteremias were not seen. This was probably related to the overall low number of hospital-acquired bacteremias due to these two organisms.

We did not identify any serious adverse effects of daily bathing with the chlorhexidineimpregnated washcloths. Serious allergic reactions have been reported with the topical use of chlorhexidine, but these reactions appear to be rare. ${ }^{23-26}$ We did not detect the emergence of MRSA or VRE isolates with high-level resistance to chlorhexidine during the study. Concern regarding increased resistance of nosocomial bacteria to biocides and disinfectants like chlorhexidine has tempered enthusiasm for wider adoption of their use in hospitals for skin antisepsis. ${ }^{27-34}$ The potential for the emergence of resistance to chlorhexidine remains a substantial concern and should be monitored over time. ${ }^{35}$

Identifying simple, cost-effective, and safe strategies for the prevention of health care-associated infection is essential. Daily bathing with chlorhex- idine-impregnated washcloths is a strategy that is relatively straightforward to implement and sustain because it does not require a substantial change from patient-bathing practices that are currently routine. We found that this intervention was associated with reductions in the rates of MRSA and VRE acquisition and hospital-acquired bloodstream infection.

The views expressed in this article are those of the authors and do not necessarily represent the official position of the Centers for Disease Control and Prevention.

Supported by a cooperative program award from the Centers for Disease Control and Prevention (5UO1C1000395-02) and Sage Products.

Dr. Climo reports receiving grant support from Sage Products. Dr. Warren reports receiving consulting fees from Centene, C.R. Bard, Cardinal Health, and Sagentia; lecture fees from $3 \mathrm{M}$ Health Care; and grant support through his institution from Cubist Pharmaceuticals and bioMérieux. Dr. Perl reports holding board memberships for Hospira and Pfizer and receiving grant support from Merck. Dr. Weinstein reports serving as an unpaid consultant for Sage Products and receiving grant support from the Foglia Family Foundation. No other potential conflict of interest relevant to this article was reported.

Disclosure forms provided by the authors are available with the full text of this article at NEJM.org.
REFERENCES

1. Grundmann H, Aires-de-Sousa M, Boyce J, Tiemersma E. Emergence and resurgence of methicillin-resistant Staphylococcus aureus as a public health threat. Lancet 2006;368:874-85.

2. Bouchillon SK, Johnson BM, Hoban DJ, et al. Determining incidence of ex tended spectrum $\beta$-lactamase producing Enterobacteriaceae, vancomycin-resistant Enterococcus faecium, and methicillinresistant Staphylococcus aureus in 38 centres from 17 countries: the PEARLS study 2001-2002. Int J Antimicrob Agents 2004;24:119-24.

3. Kreman T, Hu J, Pottinger J, Herwaldt LA. Survey of long-term-care facilities in Iowa for policies and practices regarding residents with methicillin-resistant Staphylococcus aureus or vancomycin-resistant enterococci. Infect Control Hosp Epidemiol 2005;26:811-5.

4. Streit JM, Jones RN, Sader HS, Fritsche TR. Assessment of pathogen occurrences and resistance profiles among infected patients in the intensive care unit: report from the SENTRY Antimicrobial Surveillance Program (North America, 2001). Int J Antimicrob Agents 2004;24: 111-8.

5. Furuno JP, Hebden JN, Standiford HC, et al. Prevalence of methicillin-resistant Staphylococcus aureus and Acinetobacter baumannii in a long-term acute care facility. Am J Infect Control 2008;36:468-71.

6. Siegel JD, Rhinehart E, Jackson $M$,
Chiarello L. 2007 Guideline for Isolation Precautions: preventing transmission of infectious agents in health care settings. Am J Infect Control 2007;35:Suppl 2:S65S164.

7. Warren DK, Quadir WW, Hollenbeak CS, Elward AM, Cox MJ, Fraser VJ. Attributable cost of catheter-associated bloodstream infections among intensive care patients in a nonteaching hospital. Crit Care Med 2006;34:2084-9.

8. Roberts RR, Scott RD II, Hota B, et al. Costs attributable to healthcare-acquired infection in hospitalized adults and a comparison of economic methods. Med Care 2010;48:1026-35.

9. Stone PW, Glied SA, McNair PD, et al. CMS changes in reimbursement for HAIs: setting a research agenda. Med Care 2010; 48:433-9.

10. Sipkoff M. Hospitals asked to account for errors on their watch: CMS and states may stop paying for specific hospitalacquired conditions: will health plans follow suit? Manag Care 2007;16:30, 35-7.

11. Warren DK, Cosgrove SE, Diekema DJ, et al. A multicenter intervention to prevent catheter-associated bloodstream infections. Infect Control Hosp Epidemiol 2006;27:662-9.

12. Pronovost P, Needham D, Berenholtz $S$, et al. An intervention to decrease catheter-related bloodstream infections in the ICU. N Engl J Med 2006;355:2725-32. [Erratum, N Engl J Med 2007;356:2660.]
13. Pronovost PJ, Goeschel CA, Colantuoni E, et al. Sustaining reductions in catheter related bloodstream infections in Michigan intensive care units: observational study. BMJ 2010;340:c309.

14. Vernon MO, Hayden MK, Trick WE, Hayes RA, Blom DW, Weinstein RA. Chlorhexidine gluconate to cleanse patients in a medical intensive care unit: the effectiveness of source control to reduce the bioburden of vancomycin-resistant enterococci. Arch Intern Med 2006;166:306-12. 15. Bleasdale SC, Trick WE, Gonzalez IM, Lyles RD, Hayden MK, Weinstein RA. Effectiveness of chlorhexidine bathing to reduce catheter-associated bloodstream infections in medical intensive care unit patients. Arch Intern Med 2007;167:2073-9. 16. Climo MW, Sepkowitz KA, Zuccotti $\mathrm{G}$, et al. The effect of daily bathing with chlorhexidine on the acquisition of methicillin-resistant Staphylococcus aureus, vancomycin-resistant Enterococcus, and healthcare-associated bloodstream infections: results of a quasi-experimental multicenter trial. Crit Care Med 2009; 37:1858-65.

17. Performance Standards for Antimicrobial Susceptibility Testing: Fourteenth Informational Supplement (M100-S14). Wayne, PA: National Committee for Clinical Laboratory Standards, 2004.

18. Horan TC, Andrus M, Dudeck MA. CDC/NHSN surveillance definition of health care-associated infection and cri- 
teria for specific types of infections in the acute care setting. Am J Infect Control 2008;36:309-32. [Erratum, Am J Infect Control 2008;36:655.]

19. Popovich KJ, Hota B, Hayes R, Weinstein RA, Hayden MK. Effectiveness of routine patient cleansing with chlorhexidine gluconate for infection prevention in the medical intensive care unit. Infect Control Hosp Epidemiol 2009;30:959-63. 20. Munoz-Price LS, Hota B, Stemer A, Weinstein RA. Prevention of bloodstream infections by use of daily chlorhexidine baths for patients at a long-term acute care hospital. Infect Control Hosp Epidemiol 2009;30:1031-5.

21. McDonnell G, Russell AD. Antiseptics and disinfectants: activity, action, and resistance. Clin Microbiol Rev 1999;12:14779. [Erratum, Clin Microbiol Rev 2001; 14:227.]

22. Lockhart SR, Wagner D, Iqbal N, et al. Comparison of in vitro susceptibility characteristics of Candida species from cases of invasive candidiasis in solid organ and stem cell transplant recipients: Transplant-Associated Infections Surveillance Network (TRANSNET), 2001 to 2006. J Clin Microbiol 2011;49:2404-10.

23. Sijbesma $T$, Röckmann $H$, van der Weegen W. Severe anaphylactic reaction to chlorhexidine during total hip arthroplasty surgery: a case report. Hip Int 2011;21:630-2.

24. Bringué Espuny X, Soria X, Solé E, et al. Chlorhexidine-methanol burns in two extreme preterm newborns. Pediatr Dermatol 2010;27:676-8.

25. Cheng CE, Kroshinsky D. Iatrogenic skin injury in hospitalized patients. Clin Dermatol 2011;29:622-32.

26. Liippo J, Kousa P, Lammintausta $\mathrm{K}$. The relevance of chlorhexidine contact allergy. Contact Dermat 2011;64:229-34.

27. McGann P, Kwak YI, Summers A, Cummings JF, Waterman PE, Lesho EP. Detection of qacA/B in clinical isolates of methicillin-resistant Staphylococcus aureus from a regional healthcare network in the eastern United States. Infect Control Hosp Epidemiol 2011;32:1116-9.

28. Smith K, Gemmell CG, Hunter IS. The association between biocide tolerance and the presence or absence of qac genes among hospital-acquired and community-acquired MRSA isolates. J Antimicrob Chemother 2008;61:78-84.

29. Sheng WH, Wang JT, Lauderdale TL, Weng CM, Chen D, Chang SC. Epidemiology and susceptibilities of methicillinresistant Staphylococcus aureus in Taiwan emphasis on chlorhexidine susceptibility. Diagn Microbiol Infect Dis 2009;63:309-13. 30. Alam MM, Kobayashi N, Uehara N, Watanabe N. Analysis on distribution and genomic diversity of high-level antiseptic resistance genes qacA and qacB in human clinical isolates of Staphylococcus aureus. Microb Drug Resist 2003;9:109-21.

31. Longtin J, Seah C, Siebert K, et al. Dis- tribution of antiseptic resistance genes qacA, qacB, and smr in methicillin-resistant Staphylococcus aureus isolated in Toronto, Canada, from 2005 to 2009. Antimicrob Agents Chemother 2011;55: 2999-3001.

32. Vali L, Davies SE, Lai LL, Dave J, Amyes SG. Frequency of biocide resistance genes, antibiotic resistance and the effect of chlorhexidine exposure on clinical methicillin-resistant Staphylococcus aureus isolates. J Antimicrob Chemother 2008;61:524-32.

33. Noguchi N, Nakaminami H, Nishijima S, Kurokawa I, So H, Sasatsu M. Antimicrobial agent of susceptibilities and antiseptic resistance gene distribution among methicillin-resistant Staphylococcus aureus isolates from patients with impetigo and staphylococcal scalded skin syndrome. J Clin Microbiol 2006;44:2119-25. 34. Lee AS, Macedo-Vinas M, François P, et al. Impact of combined low-level mupirocin and genotypic chlorhexidine resistance on persistent methicillin-resistant Staphylococcus aureus carriage after decolonization therapy: a case-control study. Clin Infect Dis 2011;52:1422-30.

35. Weber DJ, Rutala WA, Sickbert-Bennett EE. Outbreaks associated with contaminated antiseptics and disinfectants. Antimicrob Agents Chemother 2007;51: 4217-24.

Coppright $\odot 2013$ Massachusetts Medical Society.

JOURNAL ARCHIVE AT NEJM.ORG

Every article published by the Journal is now available at NEJM.org, beginning with the first article published in January 1812. The entire archive is fully searchable, and browsing of titles and tables of contents is easy and available to all. Individual subscribers are entitled to free 24-hour access to 50 archive articles per year Access to content in the archive is available on a per-article basis and is also being provided through many institutional subscriptions. 